\title{
Load shedding scheme based metaheuristic technique for power system controlled islanding
}

\author{
N. Z. Saharuddin', I. Zainal Abidin², H. Mokhlis ${ }^{3}$, E. F. Shair ${ }^{4}$ \\ ${ }^{1,4}$ Faculty of Electrical Engineering, Universiti Teknikal Malaysia Melaka, Durian Tunggal, Melaka, Malaysia \\ ${ }^{2}$ Department of Electrical and Electronics Engineering, Universiti Tenaga Nasional, Kajang, Selangor, Malaysia \\ ${ }^{3}$ Department of Electrical Engineering, Faculty of Engineering, University of Malaya, Kuala Lumpur, Malaysia
}

\section{Article Info}

Article history:

Received May 10, 2021

Revised Jul 21, 2021

Accepted Aug 4, 2021

\section{Keywords:}

Balanced island

MDEP load shedding technique

Minimal power imbalance

Power balance criterion

Power system islanding

\begin{abstract}
Power system-controlled islanding is one of the mitigation techniques taken to prevent blackouts during severe outage. The implementation of controlled islanding will lead to the formation of few islands, that can operate as a stand-alone island. However, some of these islands may not be balanced in terms of generation and load after the islanding execution. Therefore, a good load shedding scheme is required to meet the power balance criterion so that it can operate as a balanced stand-alone island. Thus, this paper developed a load shedding scheme-based metaheuristics technique namely modified discrete evolutionary programming (MDEP) technique to determine the optimal amount of load to be shed in order to produce balanced stand-alone islands. The developed load shedding scheme is evaluated and validated with two other load shedding techniques which are conventional EP and exhaustive search techniques. The IEEE 30-bus and 39-bus test systems were utilized for this purpose. The results proves that the load shedding based MDEP technique produces the optimal amount of loads to be shed with shortest computational time as compared with the conventional EP and exhaustive search techniques.
\end{abstract}

This is an open access article under the CC BY-SA license.

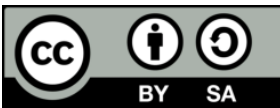

Corresponding Author:

N.Z. Saharuddin

Faculty of Electrical Engineering

Universiti Teknikal Malaysia Melaka

76100 Durian Tunggal, Melaka, Malaysia

Email: nurzawani@utem.edu.my

\section{INTRODUCTION}

Controlled islanding is executed to save the power system from severe cascading failures and blackouts [1]. One of the important criterion considered after its implementation is the power balance criterion [2]. Each island formed during islanding execution must fulfilled the power balance criterion. In other words, the total power generation in each island must be sufficient to cater the total load demand. This is very important for successful implementation of islanding execution [3]. However, there are possible to form unbalanced islands (the total load is more than the total generation) after islanding execution. In such cases, it is very important to balance the islands to avoid any further outages that could cause the islands to collapse. Thus, a load shedding scheme is needed to balance the islands by removing the necessary loads. Through this, the islands will be balanced and able to operate as stand-alone islands.

There are number of controlled islanding techniques proposed by previous researchers in recent years. Some of these techniques are ordered binary decision diagrams (OBDD) [4], [5], slow coherency approaches [6]-[9], linear programming techniques [10]-[12], metaheuristic techniques such as binary 
particle swarm optimization (BPSO) [13], angle modulated particle swarm optimization (AMPSO) [14], tabu search algorithm [15]. Detail explanation on the islanding techniques can be found in [16]. The main objective of these islanding techniques is to determine the suitable islanding strategy for a power system network. Only techniques proposed in [6]-[9] had mentioned on the utilization of UFLS load shedding scheme for islanding execution. However, detail explanation is not provided. Other techniques do not highlight the load shedding technique utilized to balance the islands if any unbalanced islands are formed after the implementation of the controlled islanding. However, achieving balanced islands in terms of generation and load balance is crucial important to ensure a successful islanding execution.

There are two common types of load shedding scheme applied in power system area, which are under voltage load shedding (UVLS) and under frequency load shedding (UFLS) schemes [17]. The UVLS is utilized to maintain the acceptable level of voltage in the power system, whereas the under frequency load shedding (UFLS) is implemented to avoid any frequency drops in the power system caused by power imbalance [18]. In this research, the developed load shedding scheme is based on the UVLS scheme. Generally, the UVLS scheme can be implemented using the exhaustive search, conventional or computational intelligence approaches. The exhaustive search is a basic technique that used to determine the optimal load to be shed [19]. It combines all the possible combinations of solutions to determine the optimal amount of loads to be shed (optimal solution). For example, if the total number of buses available for load shedding is ten, then the possible combinations of solutions are $2^{10}-1=1023$. These possible combinations will increase as the system size increases. Therefore, this technique is not relevant and impractical for large scale power systems, as it involves with huge number of possible combinations of solutions. This will cause the technique to consume longer time to find the optimal solution. For conventional technique, the fixed amount of the load is shed within the time delay setting when the undervoltage in power system is detected. However, the fixed amount is always not the best option, as in certain cases, it will end up with overshedding or undershedding the loads. This improper load shedding amount will further lead to other stability problems within the power system such as voltage collapse and blackouts [20]. Moreover, conventional load shedding scheme is not practical to be applied for today's modern and complex power system as this technique unable to provide the optimal amount of load to be shed during load shedding execution [21]. Computational intelligence approaches can provide the optimal amount of load to be shed to achieve balanced islands during controlled islanding execution. These techniques are the best, robust and adaptable for to utilize in complex, non-linear problems such as load shedding problem. Metaheuristics technique which is under computational intelligence techniques are utilized in this research to determine the optimal amount of load to be shed, in any unbalanced islands formed during controlled islanding implementation.

Several number of metaheuristic techniques have been proposed for load shedding scheme in the power system application. Among them are PSO technique [22], firefly algorithm [23], ant colony optimization [24], genetic algorithm [25], ant lion optimizer [26] and multi objective particle swarm optimization (MOPSO) [27]. All of these load shedding schemes determine the optimal load and location to be shed in order to maintain the secure power system operating state. For controlled islanding implementation, there are no detailed explanation highlighted on the load shedding scheme utilized with its implementation. Therefore, this paper proposes a new load shedding scheme based metaheuristics technique, namely modified discrete evolutionary programming (MDEP) technique to determine the optimal amount of load to be shed to obtain a balanced stand-alone islands after controlled islanding implementation. Minimal power imbalance is used as the objective function in the proposed technique.

\section{DEVELOPED TECHNIQUE}

Power generation deficit may occur in any islands formed during controlled islanding. This situation happens when the total power generation is less than the total load demand. In order to maintain the power balance criterion in each island, a load shedding scheme is required. In this paper, a load shedding scheme using modified discrete evolutionary programming (MDEP) is developed to determine the optimal amount of load to be removed in order to fulfil the power balance criterion in the islands. The general steps involved in the load shedding scheme is shown in Figure 1. Referring to Figure 1, in the first step, the power imbalance in each island is calculated using the (1),

$$
\sum P_{I M B}=\sum P_{G E N}-\left(\sum P_{L O A D}+\sum P_{L O S S}\right)
$$

where $P_{G E N}$ is the total power generation, $P_{L O A D}$ is the total load demand and $P_{L O S S}$ is the total power losses for an island. 


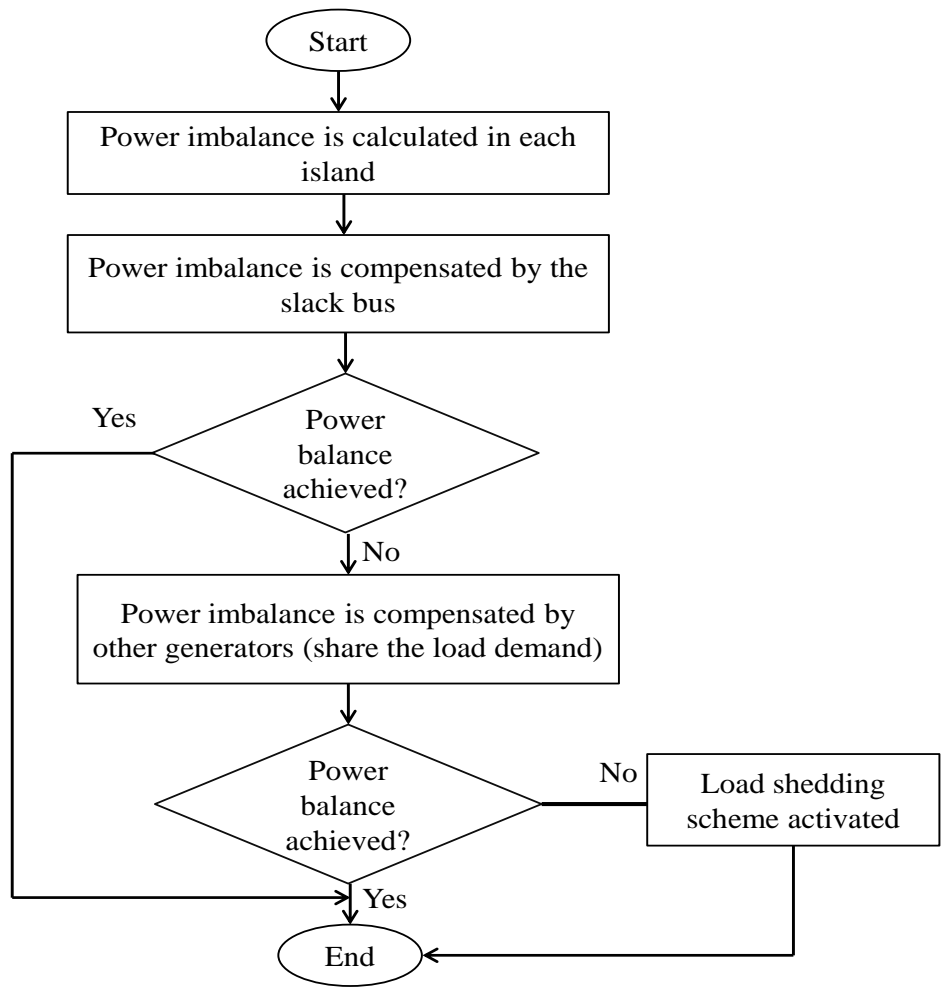

Figure 1. Steps involved in load shedding scheme

If any power imbalance is noticed in the island, the slack bus will act to compensate the power imbalance. This process will continue until the slack bus reached its maximum limit. If the power imbalance (power deficiency) still occurs, then other generators will fulfill the power imbalance. In this step, the remaining power imbalance is shared equally by the generators. This process continues until all the generators reach their maximum limit. If the power imbalance is still present after all these steps have been executed, then the proposed load shedding scheme is initiated. The implementation of the load shedding scheme will ensure the power balance criterion is met in each island formed. Details of the proposed load shedding scheme will be explained further in the following section.

\section{METHODOLOGY}

In this research, the proposed modified discrete evolutionary programming (MDEP) is chosen because the selection of the buses during load shedding action involved with discrete numbers such as bus 2 , bus 4 and bus 7 . The proposed load shedding scheme based discrete optimization technique capable to determine the optimal loads that needs to be shed in any island where power imbalance is found. Details of the proposed technique are described further in this section.

\subsection{Modified discrete evolutionary programming (MDEP) load shedding scheme}

The modified discrete evolutionary programming (MDEP) technique is used to develop the load shedding scheme in this research. The process involved in determining the optimal loads to be shed using the MDEP technique is illustrated by the flowchart shown in Figure 2. Based on Figure 2, the initial populations, $x_{p}$ are generated randomly from the available buses for load shedding either as single or different combination number of buses. The example of randomly generated initial populations is shown in Table 1.

Table 1. Example of random initial populations for MDEP load shedding scheme

\begin{tabular}{ccccc}
\hline No. of randomly chosen buses & $1^{\text {st }}$ bus & $2^{\text {nd }}$ bus & $3^{\text {rd }}$ bus & $n^{\text {th }}$ bus \\
\hline 1 & $A_{1}$ & & & \\
2 & $A_{2}$ & $A_{3}$ & & \\
3 & $A_{4}$ & $A_{5}$ & $A_{6}$ & \\
$n$ & $A_{7}$ & $A_{8}$ & $A_{9}$ & $A_{n}$ \\
\hline
\end{tabular}




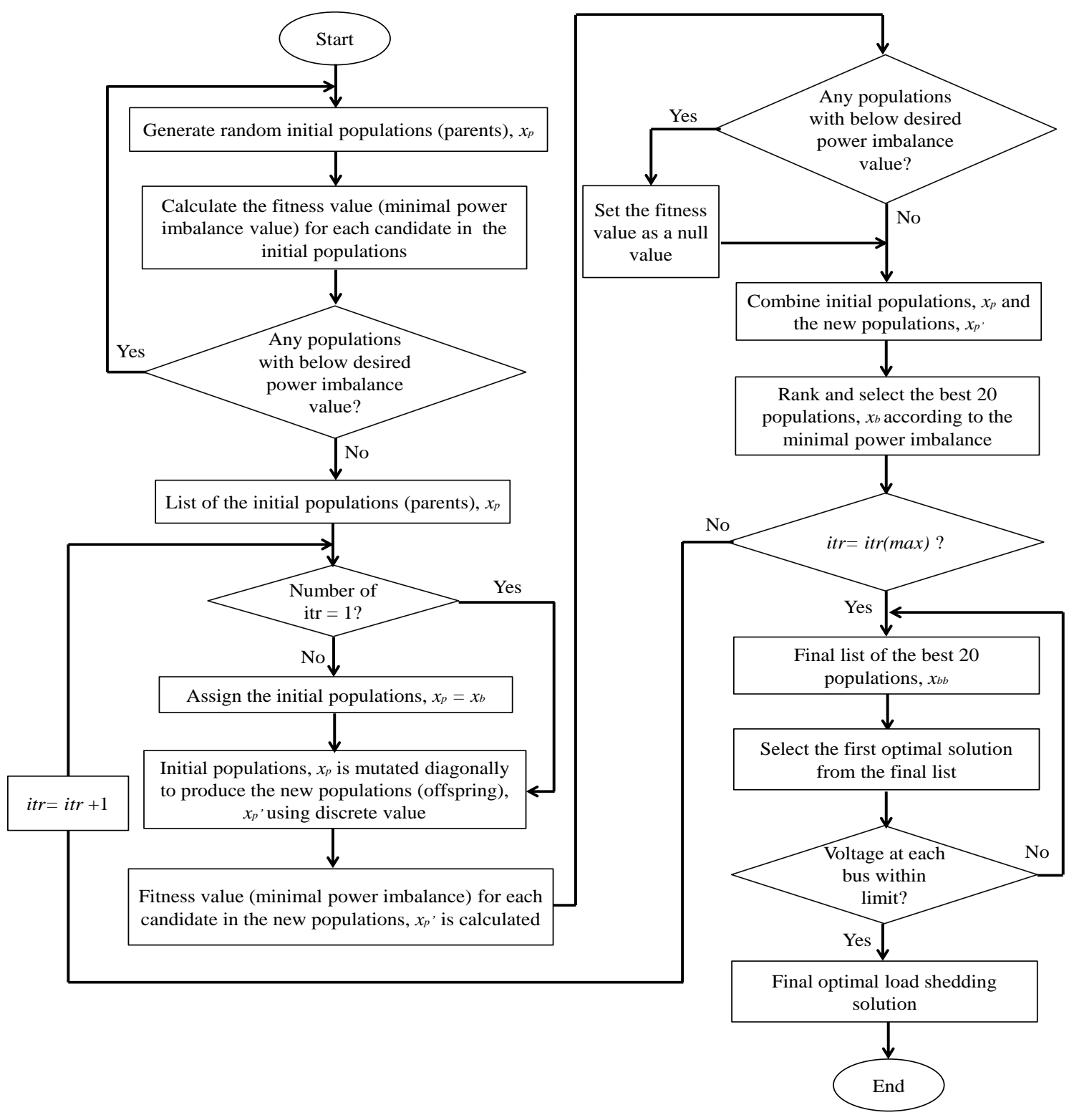

Figure 2. MDEP load shedding technique

Based on Table 1, random bus, $A_{i}$, is chosen from the total available buses for load shedding. Once the random number of initial populations is generated, the fitness function will further be calculated for each initial population. Minimal power imbalance is used as the fitness function (objective function) and Equation 1 is used to calculate this fitness function. Then, the algorithm will check if there were any populations whose fitness function value is less than the desired power imbalance value. If there is any population meet with this criterion, it will re-generate a new random initial population. Only the random initial populations which the fitness function value is same or greater than the desired power imbalance is selected as the feasible initial populations. Then, the iteration started by mutating each bus in the random initial populations, $x_{p}$ diagonally from the available buses for load shedding. Table 2 shows the example of the mutation technique applied in a diagonal form.

Table 2. Mutation process in MDEP load shedding scheme

\begin{tabular}{lllll}
\hline & Random initial population & $\mathbf{A}_{\mathbf{1}}$ & $\mathbf{A}_{\mathbf{2}}$ & $\mathbf{A}_{\mathbf{n}}$ \\
\hline 1 & $1^{\text {st }}$ bus is randomly changed & $\mathbf{r}_{\mathbf{i}}$ & $\mathrm{A}_{2}$ & $\mathrm{~A}_{\mathrm{n}}$ \\
2 & $2^{\text {nd }}$ bus is randomly changed & $\mathrm{A}_{1}$ & $\mathbf{r}_{\mathbf{i}}$ & $\mathrm{A}_{\mathrm{n}}$ \\
3 & $3^{\text {rd }}$ bus is randomly changed & $\mathrm{A}_{1}$ & $\mathrm{~A}_{2}$ & $\mathbf{r}_{\text {in }}$ \\
\hline
\end{tabular}


Referring to Table 2, each bus from the random initial population $\left(A_{1}, A_{2} \ldots A_{n}\right)$ is mutated and replaced by a random value, $r_{i n}$ from the available buses for load shedding. For example, if the initial population contains three buses, the mutation process will produce another new three mutated load shedding solution (new populations), as illustrated in Table 2. The same process will be applied for all generated initial populations. Then, the fitness function for each new population is calculated. If any population with fitness value lower than the desired power imbalance is found, the null value is set to the population, showing that the population is not a feasible load shedding solution.

Next, the combination of the new populations with the initial populations is carried out and the best 20 populations, $x_{b}$ with minimal fitness function are ranked and selected for the next iteration. The process continues until it reached the maximum number of iterations specified. Finally, the 20 final best solutions, $x_{b b}$ with minimal power imbalance are selected as the best load shedding solution.

The first load shedding solution from the final best solutions, $x_{b b}$ is selected as the optimal load shedding solution and checked if any buses violated the allowable voltage limits. The solution is considered as the optimal load shedding solution if no bus on the island violates the allowable voltage limit. Otherwise, the algorithm will select the next best solution from the final list and repeat the same process until the optimal load shedding solution is obtained for the island. By this approach, the optimal load shedding solution will be obtained for the load shedding scheme.

\section{RESULTS AND DISCUSSION}

The IEEE 30-bus and 39-bus test systems are used to demonstrate and validates the developed MDEP load shedding technique. The 30-bus test system consists of 6 generators and 41 transmission lines whereas the 39-bus test system consists of 10 generator and 46 transmission lines. Computational time and optimal amount of load to be shed are the main two criteria considered in this validation process. This work uses the MATLAB R2015a on an Intel ${ }^{\circledR}$ Core ${ }^{\mathrm{TM}}$ i7-5500U CPU at $2.40 \mathrm{GHz}$ with $8 \mathrm{~GB}$ of RAM to code the developed technique. Two case studies of controlled islanding strategy are validated in this part.

\subsection{Case I: IEEE 30-bus system}

In Case I, the controlled islanding strategy is obtained by splitting the system into two stand-alone islands based on their coherent group of generators, $\mathrm{G}_{1}=\{1,2,5,13\}$ and $\mathrm{G}_{2}=\{8,11\}$, following critical line outage of Line 1-2. The optimal islanding strategy for Case I is shown in Table 3.

Table 3. Optimal islanding strategy for case I (before load shed)

\begin{tabular}{ccccc}
\hline \multirow{2}{*}{ Islands } & Buses Info & \multicolumn{2}{c}{ Active Power (MW) } & \multirow{2}{*}{$\begin{array}{c}\text { Pofore load shed } \\
\text { Befor Imbalance }\end{array}$} \\
& & Total Pgen & Total Pload & (MW) \\
\hline Island 1 & $1-5,12-18,23$ & 327.423 & 170.400 & - \\
Island 2 & $6-11,19-22,24-30$ & 77.946 & 113.000 & 35.054 \\
\hline
\end{tabular}

According to the Table 3, it is found that Island 2 is not balance after controlled islanding execution as the total load, $\mathrm{P}_{\text {load }}$ is more than the total generation power, $\mathrm{P}_{\text {gen }}$. The power imbalance in this case is 35.054 MW. Therefore, the load shedding scheme is required to obtain the optimal amount of load to be shed in Island 2. Here, the proposed MDEP load shedding scheme is utilized for this purpose. The effectiveness of the proposed MDEP load shedding scheme is further compared and validated with two other types of load shedding scheme which are conventional EP and exhaustive search techniques. The total number of buses eligible for load shedding in Island 2- Case I is 10 which are bus 7, bus 8 , bus 10 , bus 19, bus 20, bus 21, bus 24 , bus 26 , bus 29 , bus 30 . Table 4 summarize the results obtained from this analysis.

Table 4. Result of optimal amount of load to be shed between the conventional EP, exhaustive search, and MDEP techniques for case I

\begin{tabular}{cccc}
\hline & \multicolumn{2}{c}{ Power imbalance = 35.054 MW } & Computational time (sec) \\
Technique & Optimal amount of load to be shed (MW) & Bus(es) & Cons \\
\hline Conventional EP & 35.7 & $19,21,24$ & 71.0422 \\
Exhaustive search & 35.1 & $19,20,21,26,29$ & 4.9869 \\
MDEP & 35.1 & $19,20,21,26,29$ & 4 \\
\hline
\end{tabular}


Referring to Table 4, the exhaustive search and MDEP load shedding techniques capable to obtain a better optimal amount of load to be shed which are 35.1 MW as compared to conventional EP technique that obtained 35.7 MW. The conventional EP unable to produce the best optimal value due to the usage of Gaussian function which result in small changes during mutation process in the conventional EP. Although the exhaustive search able to produce the same optimal value as the MDEP load shedding technique, it requires a longer computational time of 71.0422 seconds to produce the optimal amount of load to be shed compared with the MDEP technique which only requires 4.9869 seconds to produce the same optimal value. The exhaustive search generally combines all the possible combinations of available buses in the system to determine the optimal solution. The longer computational time is required for this technique as the system size increases (more possible combinations of solutions). Therefore, the proposed MDEP load shedding technique is the best load shedding scheme as it can determine the optimal load shedding amount with shortest time compared to other techniques illustrated in Table 4.

The MDEP load shedding is then utilized to shed the optimal amount of load in Island 2 in order to meet the power balance criterion in the island. Table 5 shows the optimal islanding strategy after load shedding execution (35.100 MW) in Island 2, where the total generation power, $\mathrm{P}_{\text {gen }}(78.517 \mathrm{MW})$ is now more than the total load, $\mathrm{P}_{\text {load }}(77.900 \mathrm{MW})$. Thus, Island 2 can now operate as a balanced stand-alone island successfully.

Table 5. Optimal islanding strategy for case I (after load shed)

\begin{tabular}{ccccc}
\hline \multirow{2}{*}{ Islands } & \multirow{2}{*}{ Buses Info } & \multicolumn{2}{c}{ Active Power (MW) } & \multirow{2}{*}{ After load shed } \\
& & Total Pgen & Total Pload & (MW) \\
\hline Island 1 & $1-5,12-18,23$ & 189.446 & 170.400 & - \\
Island 2 & $6-11,19-22,24-30$ & 78.517 & 77.900 & 35.100 \\
\hline
\end{tabular}

\subsection{Case II: IEEE 39-bus system}

In Case II, the controlled islanding strategy is obtained by splitting the system into two stand-alone islands based on their coherent group of generators, $G_{1}=\{30,31,32,37,38,39\}$ and $G_{2}=\{33,34,35,36\}$, following critical line outage of Line 13-14. Table 6 shows the optimal islanding strategy for Case II.

Table 6. Optimal islanding strategy for case II (before load shed)

\begin{tabular}{ccccc}
\hline \multirow{2}{*}{ Islands } & \multirow{2}{*}{ Buses Info } & \multicolumn{2}{c}{ Active Power (MW) } & \multirow{2}{*}{ Power Imbalance } \\
& & Total Pgen & Total Pload & (MW) \\
\hline Island 1 & $1-15,18,25,26,28-32,37-39$ & 4021.668 & 4134.130 & 112.462 \\
Island 2 & $16-17,19-24,27,33-36$ & 2134.198 & 2120.100 & - \\
\hline
\end{tabular}

Referring to the Table 6 , it is found that Island 1 is not balance after islanding execution as the total load, $\mathrm{P}_{\text {load }}$ is more than the total generation power, $\mathrm{P}_{\mathrm{gen}}$. The power imbalance in this case is $112.462 \mathrm{MW}$. Therefore, a load shedding scheme is required to obtain the optimal amount of load to be shed in Island 1. Here, the proposed MDEP load shedding scheme is utilized for this purpose. The effectiveness of the proposed MDEP load shedding scheme is further compared and validated with two other types of load shedding scheme which are conventional EP and exhaustive search techniques. The total number of buses eligible for load shedding in Island 1 - Case II is 15 which are bus 1 , bus 3 , bus 4 , bus 7, bus 8 , bus 9 , bus 12 , bus 15 , bus 18 , bus 25 , bus 26 , bus 28 , bus 29 , bus 31 , bus 39 . Table 7 summarize the results obtained from this analysis.

Table 7. Result of optimal amount of load to be shed between the conventional EP, exhaustive search, and MDEP algorithms for case II

\begin{tabular}{cccc}
\hline & \multicolumn{3}{c}{ Power imbalance $=112.462 \mathrm{MW}$} \\
\hline Technique & Optimal amount of load to be shed (MW) & Bus(es) & Computational time (sec) \\
Conventional EP & 115.33 & $1,12,31$ & 8.8957 \\
Exhaustive search & 112.63 & $1,9,12$ & 89486.7264 \\
MDEP & 112.63 & $1,9,12$ & 3.1935 \\
\hline
\end{tabular}


According to Table 7, similar in Case I, the exhaustive search and MDEP load shedding techniques are capable to obtain a better optimal amount of load to be shed which are $112.63 \mathrm{MW}$ as compared to conventional EP technique that obtained 115.33 MW. The usage of Gaussian function is the main contributor of inaccurate optimal value obtained in the conventional EP. Although the exhaustive search can produce the same optimal value as the MDEP technique, however, it takes a longer computational time of 89486.7264 seconds to produce the optimal solution compared to the MDEP technique which only takes 3.1935 seconds to produce the same optimal solution. This is because the exhaustive search technique combines all the possible combinations of available buses in the system to determine the optimal solution which increases the computational time as the system size increases. Therefore, both conventional EP and exhaustive search techniques are not suitable to be used as the load shedding scheme in this research. Hence, the proposed MDEP load shedding technique is proven to be the best load shedding scheme as it can determine the optimal load shedding amount with the shortest computational time compared to conventional EP and exhaustive search techniques.

The MDEP load shedding then utilized to shed the optimal amount of load in Island 1 in order to meet the power balance criterion in that island. Table 8 shows the optimal islanding strategy after load shedding execution $(112.630 \mathrm{MW})$ in Island 1 , where the total generation power, $\mathrm{P}_{\text {gen }}(4063.821 \mathrm{MW})$ is now more than the total load, $\mathrm{P}_{\text {load }}(4021.50 \mathrm{MW})$. Thus, Island 1 can now operate as a balanced stand-alone island successfully.

Table 8. Optimal islanding strategy for case II (after load shed)

\begin{tabular}{ccccc}
\hline \multirow{2}{*}{ Islands } & \multirow{2}{*}{ Buses Info } & \multicolumn{2}{c}{ Active Power (MW) } & \multirow{2}{*}{ After load shed } \\
& & Total Pgen & Total Pload & (MW) \\
\hline Island 1 & $1-15,18,25,26,28-32,37-39$ & 4063.821 & 4021.50 & 112.630 \\
Island 2 & $16-17,19-24,27,33-36$ & 2134.198 & 2120.100 & - \\
\hline
\end{tabular}

\section{CONCLUSION}

This paper proposed a new MDEP based load shedding scheme for controlled islanding application. The purpose of this scheme is to determine the optimal amount of load to be shed in order to achieve balanced stand-alone islands after islanding implementation. The effectiveness of the proposed scheme is validated with two different techniques which are conventional EP and exhaustive search techniques, using IEEE 30-bus and 39-bus test systems. The results proved that proposed MDEP load shedding scheme capable on determining the optimal amount of load to be shed with lower computational time as compared to conventional EP and exhaustive search techniques as presented in Case I and Case II, respectively. As such, MDEP load shedding scheme is proposed as the best scheme to be implemented after the controlled islanding execution, to fulfil the power balance criterion in any islands where load shedding is required.

\section{ACKNOWLEDGEMENTS}

The author would like to express her appreciation to Ministry of Higher Education Malaysia (MOHE) via FRGS Grant (FRGS/1/2018/TK07/UNITEN/01/1) to fund this research. In addition, the author would like to thank Universiti Teknikal Malaysia Melaka (UTeM) and Universiti Tenaga Nasional (UNITEN) for their support in this research.

\section{REFERENCES}

[1] N. Z. Saharuddin, I. Z. Abidin, and H. Mokhlis, "Discrete Evolutionary Programming for Network Splitting Strategy: Different Mutation Technique," Indonesian Journal of Electrical Engineering and Computer Science (IJEECS), vol. 12, no. 1, pp. 261-268, 2018, doi:10.11591/ijeecs.v12.i1.pp261-268.

[2] M. Goubko and V. Ginz, "Improved Spectral Clustering for Multi-Objective Controlled Islanding of Power Grid," Energy Syst., vol. 10, no. 1, pp. 1-36, 2017, doi: 10.1007/s12667-017-0240-1.

[3] Esmaeilian and M. Kezunovic, "Prevention of power grid blackouts using intentional islanding scheme," IEEE Trans. Ind. Appl., vol. 53, no. 1, pp. 622-629, 2017, doi:10.1109/TIA.2016.2614772.

[4] Q. Zhao, K. Sun, D. Zheng, J. Ma, and Q. Lu, "A study of system splitting strategies for island operation of power system: a two-phase method based on OBDDs," IEEE Trans. Power Syst., vol. 18, no. 4, pp. 1556-1565, 2003, doi:10.1109/TPWRS.2003.818747.

[5] K. Sun, D. Z. Zheng, and Q. Lu, "Splitting strategies for islanding operation of large-scale power systems using OBDD-based methods," IEEE Trans. Power Syst., vol. 18, pp. 912-923, 2003, doi:10.1109/TPWRS.2003.810995. 
[6] H. You, V. Vittal, and X. Wang, "Slow Coherency-Based Islanding," IEEE Trans. Power Syst., vol. 19, no. 2, pp. 483-491, 2004, doi:10.1109/TPWRS.2003.810995.

[7] X. Wang, and V. Vittal, "System Islanding Using Minimal Cutsets with Minimum Net Flow," in Power System Conference and Exposition, 2004, pp. 1-6, doi:10.1109/PSCE.2004.1397589.

[8] B. Yang, V. Vittal, and G. T. Heydt, "A Novel Slow Coherency Based Graph Theoretic Islanding Strategy," IEEE Trans. Power Syst., pp. 1-7, 2007, doi:10.1109/PES.2007.386173.

[9] J. Q. Tortos and V. Terzija, "Controlled islanding strategy considering power system restoration constraints," IEEE Power Energy Soc. Gen. Meet., pp. 1-8, 2012, doi:10.1109/PESGM.2012.6344599.

[10] P. A. Trodden, W. A. Bukhsh, A. Grothey, and K. I. M. Mckinnon, "MILP Formulation for Islanding of Power Networks," IEEE Trans. POWER Syst., vol. 45, no. 1, pp. 1-8, 2014, doi:10.1016/j.ijepes.2012.09.018.

[11] P. A. Trodden, W. A. Bukhsh, S. Member, A. Grothey, and K. I. M. Mckinnon, "Optimization-based Islanding of Power Networks using Piecewise Linear AC Power Flow,” IEEE Trans. Power Syst., vol. 29, no. 3, pp. 1212-1220, 2014, doi:10.1109/TPWRS.2013.2291660.

[12] P. Demetriou, A. Kyriacou, E. Kyriakides, and C. Panayiotou, "Applying Exact MILP Formulation for Controlled Islanding of Power Systems," in 51st International Universities Power Engineering Conference (UPEC), 2016, doi:10.1109/UPEC.2016.8114001.

[13] W. Liu, L. Liu, and D. A.Carter, "Binary Particle Swarm Optimization Based Defensive Islanding Of Large Scale Power Systems," Int. J. Comput. Sci. Appl., vol. 4, no. 3, pp. 69-83, 2007.

[14] L. Liu, W. Liu, D. A. Cartes, and I. Chung, "Slow coherency and Angle Modulated Particle Swarm Optimization based islanding of large-scale power systems," Adv. Eng. Informatics, vol. 23, pp. 45-56, 2009, doi:10.1109/IJCNN.2007.4371280.

[15] F. Tang, H. Zhou, Q. Wu, H. Qin, J. Jia, and K. Guo, "A Tabu Search Algorithm for the Power SystemIslanding Problem," Energies, vol. 8, no. 10, pp. 11315-11341, 2015, doi:10.3390/en81011315.

[16] N. Z. Saharuddin, I. Z. Abidin, H. Mokhlis, and K. Naidu, "Intentional Islanding Methods as Post Fault Remedial Action : A Review," Indonesian Journal of Electrical Engineering and Computer Science (IJEECS), vol. 12, no. 1, pp. 182-192, 2018, doi:10.11591/ijeecs.v12.i1.pp182-192.

[17] M. Majidi, M. Aghamohammadi, and M. Manbachi, "New design of intelligent load shedding algorithm based on critical line overloads," Turk. J. Elec. Eng. Comp. Sci., vol. 22, pp. 1395-1409, 2014, doi:10.3906/elk-1112-52.

[18] M. Lu, W. A. ZainalAbidin, T. Masri, D. H. Lee, and S. Chen, "Under-Frequency Load Shedding ( UFLS ) Schemes - A Survey,” Int. J. Appl. Eng. Res., vol. 11, no. 1, pp. 456-472, 2016.

[19] J. Nievergelt, "Exhaustive search, combinatorial optimization and enumeration: Exploring the potential of raw computing power," Lect. Notes Comput. Sci. (including Subser. Lect. Notes Artif. Intell. Lect. Notes Bioinformatics), vol. 1963, pp. 18-35, October 2000, doi:10.1007/3-540-44411-4_2.

[20] C. Adewole, R. Tzoneva, and A. Apostolov, "Adaptive under-voltage load shedding scheme for large interconnected smart grids based on wide area synchrophasor measurements," IET Gener. Transm. Distrib., pp. 1957-1968, 2016, doi:10.1049/iet-gtd.2015.1250.

[21] R. Kanimozhi, K. Selvi, and K. M. Balaji, "Multi-objective approach for load shedding based on voltage stability index consideration," Alexandria Eng. J., vol. 53, no. 4, pp. 817-825, 2014, doi:10.1016/j.aej.2014.09.005.

[22] F. HE, Y. Wang, K. W. Chan, Y. Zhang, and S. Mei, "Optimal load shedding strategy based on particle swarm optimization," in 8th International Conference on Advances in Power System Control, Operation and Management (APSCOM 2009), 2009, pp. 1-6, doi:10.1049/cp.2009.1795.

[23] X. Yang, "Firefly Algorithms for Multimodal Optimization," Stoch. Algorithms Found. Appl. SAGA, vol. 5792, pp. 169-178, 2009, doi:10.1007/978-3-642-04944-6_14.

[24] C. Church, W. G.Morsi, C. P. Diduch, M. E. El-Hawary, and L. Chang, "Voltage Collapse Detection Using Ant Colony Optimization for Smart Grid Applications," in 2010 IEEE Electrical Power \& Energy Conference, 2010, pp. 1-5, doi:10.1109/EPEC.2010.5697185.

[25] F. M. Gatta, A. Geri, S. Lauria, M. Maccioni, and P. Masato, "Power system adequacy: An efficient procedure based on genetic algorithms," in 2009 IEEE Bucharest PowerTech, 2009, doi:10.1109/PTC.2009.5282192.

[26] Z. Mat Yasin, H. Mohamad, I. Na. Sam'on, N. Ab Wahab, and N. A. Salim, "Optimal Undervoltage Load Shedding using Ant Lion Optimizer," Int. J. Simul. Syst. Sci. Technol., vol. 10. no. 55, pp. 47.1-47.16, 2018, doi:10.4314/jfas.v10i5s.35.

[27] A. Hafez, A. Y. Hatata, and A. Y. Abdelaziz, "Multi-Objective Particle Swarm for Optimal Load Shedding Remedy Strategies of Power System," Electr. Power Components Syst., vol. 47, no. 18, pp. 1651-1666, 2019, doi:10.1080/15325008.2019.1689454. 


\section{BIOGRAPHIES OF AUTHORS}
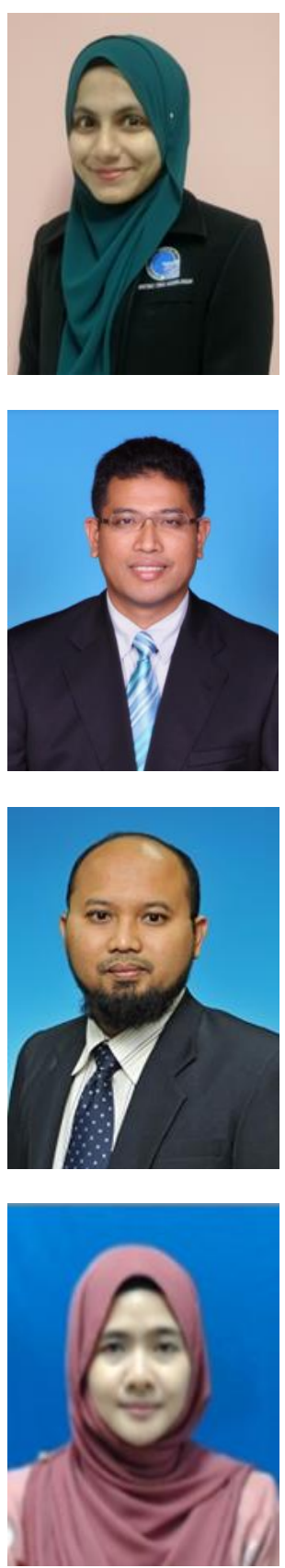

Nur Zawani Saharuddin received her Diploma in Electrical Engineering and B.Eng. in Electrical Engineering (Industrial Power) from Universiti Teknikal Malaysia Malaysia, Malaysia in 2005 and 2008, respectively. Then, she received her M.Eng. in Electrical Engineering from University of Malaya (UM) and the Ph.D. degree from Universiti Tenaga Nasional, Malaysia, in 2020. Currently she is a lecturer in the Faculty of Electrical Engineering, Universiti Teknikal Malaysia Malaysia, Malaysia. Her research interests are in the area related to power system analysis, power system islanding, and renewable energy.

Izham Zainal Abidin received his Ordinary National Diploma in Engineering from Coventry Technical College in 1994. Then, he received his B.Eng. in Electrical Engineering from University of Southampton, UK, in 1997 and Ph.D. degree in Electrical Engineering (Power) from Strathclyde University, U.K., in 2002. Currently he is a Professor in the Department of Electrical and Electronics Engineering, Universiti Tenaga Nasional, Malaysia. He is also a Senior Member (80617173) of IEEE Power and Energy Society. His research interests are in the area related to protection, voltage stability, renewal energy impact, re-configurable grids and power system stability studies.

Hazlie Mokhlis (Senior Member, IEEE) received the B.Eng. and M.Eng.Sc. degrees in electrical engineering from the University of Malaya (UM), Malaysia, in 1999 and 2002, respectively, and the Ph.D. degree from The University of Manchester, Manchester, U.K., in 2009. He is currently a Professor with the Department of Electrical Engineering, University of Malaya (UM), and also the Head of the UM Power and Energy System (UMPES) research. His research interests include fault location, distribution automation, power system protection, and renewable energy. He is also a Chartered Engineer in the U.K. and a Professional Engineer in Malaysia.

Ezreen Farina Shair received her B.Eng in Electrical - Control \& Instrumentation and M.Eng in Electrical - Mechatronics \& Automatic Control from the Universiti Teknologi Malaysia (UTM) in 2009 and 2011, respectively. Then, she received her Ph.D. in Electronics Engineering from the Universiti Putra Malaysia in 2019. Currently, she is a senior lecturer in Universiti Teknikal Malaysia Melaka and the secretary for IEEE-EMBS Malaysia Chapter. From 2018 until now, she is one of the technical editorial board for the International Journal of Human and Technology Interaction. Her research interests are on biomedical signal processing, machine learning, and IoT. 